\section{In teraction effects of chlorpromazine and reticular stimulation on visual attention behavior in rats*}

\author{
MONA ELIASSON† and CONAN KORNETSKY \\ Boston University School of Medicine, Boston, Mass. 02118
}

The effects of electrical stimulation of the reticular formation and of chlorpromazine on a visual attention task in the rat were studied. Stimulation at low intensities tended to improve the performance of the animals; however, at higher intensities of stimulation or after chlorpromazine, performance was significantly impaired. Chlorpromazine given with stimulation resulted in performance levels not different from that seen after saline alone. In one animal in which electrodes were not in the reticular formation, chlorpromazine did not reverse the effects of stimulation. These results support the hypothesis that the arousal-reducing properties of chlorpromazine are mediated by its action on the reticular formation.

In a previous study (Kornetsky \& Eliasson, 1969), we examined by means of an animal model the notion that impaired attention behavior in some schizophrenic patients is the result of a state of central hyperarousal and that the therapeutic action of chlorpromazine is to reduce this putative state. We found that electrical stimulation of the reticular formation in rats caused a specific impairment of attention behavior-an increase in the number of omitted responses to an auditory signal-and that this deficit could be reversed by chlorpromazine at a dose that by itself impairs this task. The aims of the present study were to replicate these findings on a similar task using visual stimuli and to study the effects of more than one dose of chlorpromazine on this performance at different levels of arousal produced by electrical stimulation of the reticular formation.

$$
\text { METHOD }
$$

Four rats of the Charles River CDF strain, maintained at approximately $80 \%$ of ad lib weight, served as Ss. Each rat was implanted with two bipolar stainless steel electrodes, insulated except for the tips and having a diameter of 0.010 in. These electrodes were placed bilaterally, aiming for the mesencephalic reticular formation, with the aid of a stereotaxic instrument. The coordinates were: $2 \mathrm{~mm}$ anterior to the lambda, $2 \mathrm{~mm}$ lateral to the midline, and $5.5 \mathrm{~mm}$ below the top of the skull. At the time of surgery, the animals were about 120 days old and weighed $260-290 \mathrm{~g}$.

Training on the attention task was

* This research was supported by a grant from the National Institute of Mental Health, MH 12568, and Research Scientist Award (C.K.) MH 1759.

†Present address: University of Uppsala. Uppsala, Sweden. begun after recovery from surgery. The task was performed in a standard operant conditioning chamber, which also was used in the testing procedures. After acquisition of the barpressing response with food as a reward, the conditioned stimulus (CS) was introduced. The CS consisted of a lower right. light, located on the testing-box wall above the bar, which flashed once for $1 \mathrm{sec}$ at the start of the training and then gradually was shortened over training sessions to the final 0.15 -sec duration. One depression of the bar within 5 sec of the onset of the CS was rewarded with a food pellet. The light was presented at irregular intervals, on the average of every $1.5 \mathrm{sec}$, counted from the end of the 5 -sec period to the presentation of the next CS, at the start of the training. This interval was increased over sessions until the final condition of $7.5 \mathrm{sec}$. A barpress at any other time but once during the available response time was counted as an incorrect response and punished with a 15-sec "time-out" period and postponement of new CS presentations. Failure to respond had no consequences, except for loss of reward.

Each session consisted of a short preliminary period with 25 trials, followed by a regular $45 \cdot \mathrm{min}$ testing session. On treatment days, the Ss were discontinued for that day if, during the initial 25 -trial period, their performance fell below $80 \%$ correct and/or above $25 \%$ incorrect responses.

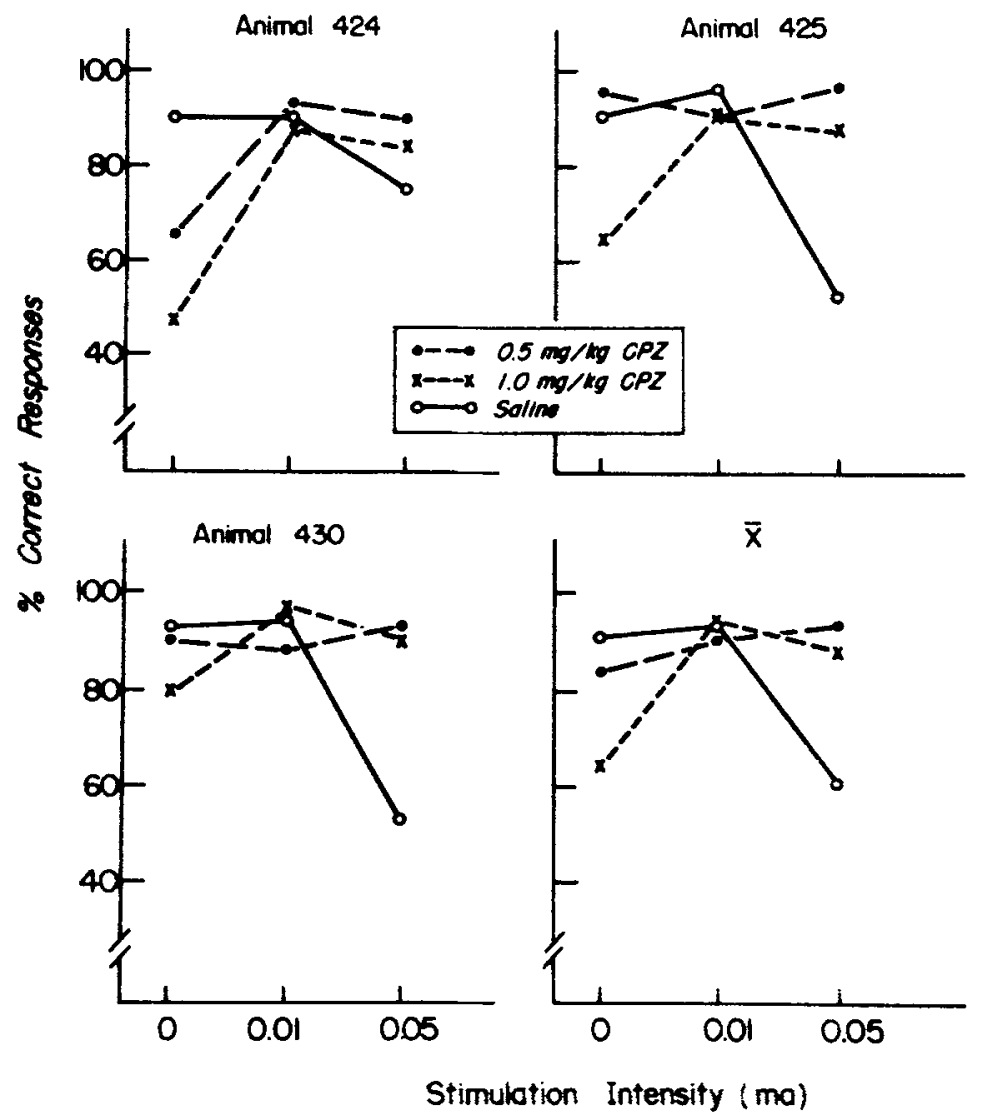

Fig. 1. The effects of chlorpromazine (CPZ) on percent correct responses as a function of level of stimulation (electrodes in the mesencephalic reticular formation) for each of three animals. Mean percent correct responses shown in 

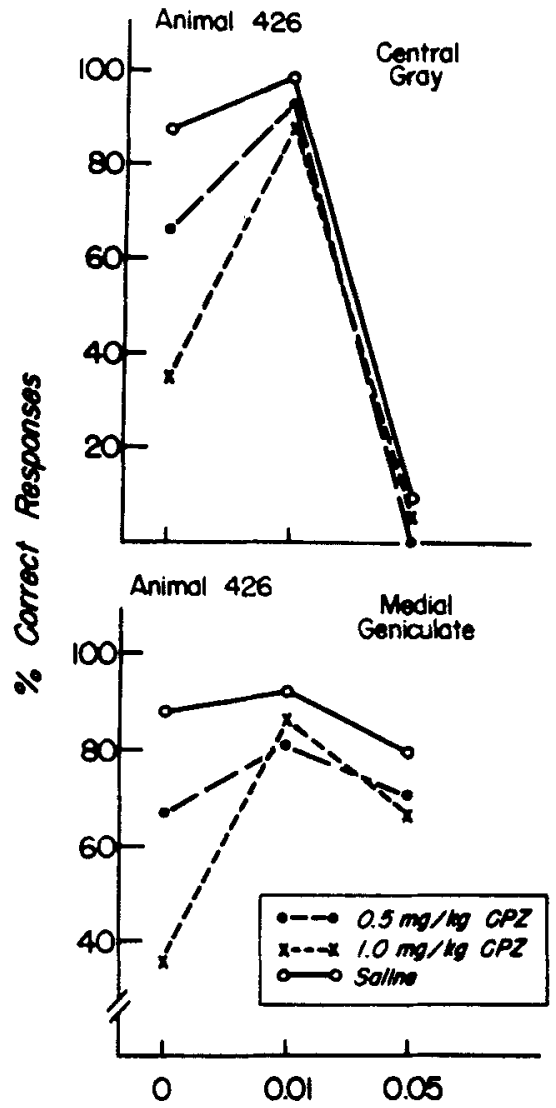

Stimulation Imtensity (ma)

Fig. 2. The effects of chlorpromazine (CPZ) on percent correct responses as a function of level of stimulation for animal with electrodes in the central gray and the medial geniculate.

Only the data collected during the 45-min session was used in the final analysis. Measures of the performance were percentage correct responses calculated in relation to the total number of possible correct responses, i.e., the number of CS presentations during the session and percentage incorrect responses also calculated in relation to the number of $\mathrm{CS}$ presentations.

Treatment in the form of electrical stimulation was delivered during the intertrial intervals immediately before, but never overlapping with, the CS presentation. Stimulation from a constant-current stimulator consisted of biphasic rectangular waves at $0.01-\mathrm{mA}$ or $0.05-\mathrm{mA}$ intensity, $100-\mathrm{Hz}$ frequency, and 0.2 -msec pulse duration. Pulse train was $500 \mathrm{msec}$. Chlorpromazine hydrochloride was administered in two doses: $0.5 \mathrm{mg} / \mathrm{kg}$ and $1.0 \mathrm{mg} / \mathrm{kg} \mathrm{IP}$ immediately after the preliminary 25-trial period. After the injection, the animals were returned to their home cages for
$15 \mathrm{~min}$, after which testing took place. Each animal served as his own control (saline), receiving both stimulation intensities, both doses of the drug, and all combinations twice. One electrode placement area was stimulated first with the low intensity, the other with the high intensity. The order was alternated for subsequent treatment days. On any single test day, stimulation was administered through only one of the electrodes. Also, the site of stimulation was alternated on subsequent days. Treatments were given twice a week if the performance showed complete recovery from the previous treatment session.

At the completion of testing, the animals were sacrificed and perfused with saline and formalin. The brains were removed, sectioned, and stained for histological examination.

\section{RESULTS}

In one $S$, the electrode missed the intended placements; those results are not included in the group data but will be discussed separately. Since only percentage correct responses showed any relationship with the reticular stimulation and chlorpromazine treatments, only that measure is discussed here.

The results for each of the three animals with electrodes in the mesencephalic reticular formation are shown in Fig. 1. An analysis of variance for repeated measures (Winer, 1962) yielded an $F$ ratio of $4.62(p<.01)$ for treatments. Further analysis by means of Fisher's LSD procedure (Fryer, 1966 ) indicated that performance after $1.0 \mathrm{mg} / \mathrm{kg}$ of chlorpromazine or $0.05-\mathrm{mA}$ stimulation was significantly different from all other treatments $(p<.01)$. When the animals were given $1.0 \mathrm{mg} / \mathrm{kg}$ of chlorpromazine and stimulation at the $0.05-\mathrm{mA}$ intensity, performance was not significantly different from that seen after saline alone

Histology showed the tips of the electrodes to be located in the dorsolateral mesencephalic reticular formation immediately ventral to the superior colliculus in three of the animals. The fourth $S$ had one electrode in the lateral central gray and one in the nucleus marginalis of the medial geniculate.

In the animal in which the electrodes were not in the mesencephalic reticular formation, the effects of chlorpromazine and stimulation were somewhat different (Fig. 2). Although stimulation of the central gray did cause a marked decrease in errors, it also markedly increased incorrect responses which were unaltered in animals stimulated in the reticular formation. Also, at levels of stimulation that impaired performance, chlorpromazine did not reverse the obtained impairment. Stimulation of the medial geniculate at $0.05 \mathrm{~mA}$ only slightly impaired the performance of the animal. Chlorpromazine at this level of stimulation did not reverse the effect; however, performance after $1.0 \mathrm{mg} / \mathrm{kg}$ of chlorpromazine and $0.05-\mathrm{mA}$ stimulation was better than performance at this dose of chlorpromazine alone.

\section{DISCUSSION}

The results reported here confirm and extend our previous findings (Kornetsky \& Eliasson, 1969). The impairment of attention performance by a high arousal level, as produced by electrical stimulation of the reticular formation, can be reversed by chlorpromazine.

Our results are in general agreement with those of Wilson \& Radloff (1967). These workers tested the effects of reticular stimulation on two operant schedules of reinforcement and found significant improvement only on the easier task and significant impairment on the more difficult task. Although not reaching an acceptable level of significance, we did find slight improvement in performance in two and no change in one of the reticular stimulation animals at the $0.01-\mathrm{mA}$ level. The procedure that we used in which all animals were performing at $89 \%$ correct or better allowed little room for additional improvement. Wilson and Radloff's two schedules probably required different basal levels of arousal during performance; we have found that much higher intensities of reticular stimulation than those used in the present study are needed to produce significant effects on conditioned avoidance response (Eliasson \& Kornetsky, 1970).

The failure of chlorpromazine to block the behavioral effects of stimulation of the central gray or the medial geniculate strongly supports the hypothesis that the arousal-reducing properties of chlorpromazine are mediated by its actions on the reticular formation.

\section{REFERENCES}

ELIASSON, M.. \& KORNETSKY, C. Effects of reticular stimulation and chlorpromazine on avoidance behavior in the rat. Federation Proceedings, 1970, 29, 486 (Abstract).

FRYER, H. C. Concepts and methods of experimental statistics. Boston: Allyn \& Bacon, 1965

KORNETSKY, C., \& ELIASSON, M. Reticular stimulation and chlorpromazine: An animal model for schizophrenic overarousal. Science, 1969. $165,1273-1274$.

WILSON, G. T. \& RADLOFF, W.P. Degree of arousal and performance: Effects of reticular stimulation on an operant task. Psychonomic Science, 1967, 7, 13-14.

WINER, B. J. Statistical principles in experimental design. New York: McGraw-Hill, 1962 . 